\title{
Schnipselbuch für Schulleiter*innen
}

DOI: https://doi.org/10.53349/sv.2021.i3.a145

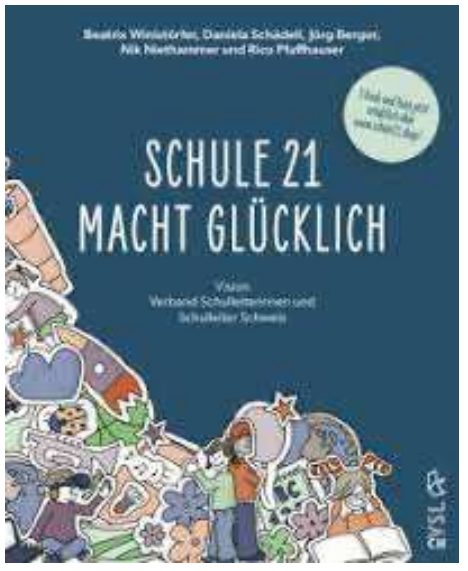

Beatrix Winistörfer, Daniela Schädeli, Jörg

Berger, Nik Niethammer und Rico Pfaffhauser

Schule 21 macht glücklich

Vision Verband Schulleiterinnen und

Schulleiter Schweiz

Kalt Medien, Zug 2021

ISBN 978-3-85761-335-7

Können Sie sich noch an die Schnipselbücher erinnern, welche in der Zeit vor dem Internet für viele Lehrkräfte unerlässlich waren? Es waren Bücher, gefüllt mit Bildern und Zeichnungen, welche für Unterrichtsmaterialien zur Illustration verwendet werden konnten. Heute werden Bilder gegoogelt, früher wurde das Schnipselbuch konsultiert.

Das vom Verband der Schulleiterinnen und Schulleiter der Schweiz herausgegebene Buch erinnert mich an diese Schnipselbücher. Diese Assoziation wird durch die wunderbaren Zeichnungen von Beatrix Winistörfer geweckt, welche das Buch lebendig und fröhlich machen und mitverantwortlich sind, dass das Buch so gar nicht nach einem Fachbuch aussieht. Aber auch die verschiedenen Kurztexte aus der Praxis von Schweizer Schulleitenden wirken wie bunte Schnipsel. Sie geben eindrucksvolle Einblicke in Schulleitungsbüros und machen durch die Textbeiträge heutiges Schulleitungshandeln in seiner Vielfalt sichtbar. 


\section{Bunte Vielfalt an Innovationen}

Schule 21 macht glücklich ist ein Buch, welches nicht von vorne nach hinten gelesen wird. Die Lesenden haben die Möglichkeit zwischen Kurztexten, welche zwei bis vier Seiten lang sind, hin und her zu hüpfen. Zur Orientierung sind diese Texte in acht Kategorien unterteilt:

- Leben und Lernen im 21. Jahrhundert

- Lernen im Alltagsleben mit allen Sinnen

- Den (digitalen) Raum nutzen - ethisch und verantwortungsvoll

- Selbstgestaltungskompetenz von Schülerinnen und Schüler

- Selbstgestaltung und Kollaboration unter Mitarbeitenden

- Das Selbstverständnis der Schule 21 als Teil eines Netzwerkes

- Hauptakteure erhalten die Beachtung, die ihnen zusteht

- Schule 21 bedeutet ...

Das Buch ist als „Book Sprint" entstanden: Der Schweizer Verband der Schulleiterinnen und Schulleiter hat seine Mitglieder und andere Interessierte dazu aufgerufen, kurze Texte über innovative Elemente ihres Alltags für ein gemeinsames Buch einzureichen. Die eingelangten Beiträge wurde von den Herausgeber*innen gesichtet, begutachtet und zum vorliegenden Buch zusammengestellt. Eine aus meiner Sicht tolle Idee, das Wissen und Können der teilnehmenden Schulleiter*innen im Sinne des Von- und Miteinanderlernens zu nutzen und sichtbar zu machen.

\section{Agilität als Führungsprinzip}

Im Rahmen dieser Rezension ist es nicht möglich, auf jeden einzelnen Beitrag im Detail einzugehen. Ich habe mich deshalb entschieden, nur einen Text, „Agilität als Führungsprinzip“ von Marco Schraner, aufzugreifen und kurz zu disktieren. Dies auch im Sinne der Metapher des Schnipselbuches, welches die Leser*innen dazu einlädt, zwischen den Texten zu springen und sich anspringen zu lassen.

Sehr kompakt auf zwei Seiten beschreibt Marco Schraner, seit zehn Jahren Schulleiter, wie er an seiner Schule OneNote-Tagebücher als Instrument zur Verbesserung der Zusammenarbeit und Schulentwicklung verwendet und wie diese als Element einer agilen Führung eingesetzt werden können. Schraner führt aus, dass mit dem flächendeckenden Einsatz von OneNoteNotizbüchern einerseits „durch den Wegfall der Teamsitzungen grundsätzlich sehr viel Zeit für das Entwickeln von Neuem frei wurde" (S. 102) und andererseits eine hohe Transparenz zwischen den Lehrer*innen hergestellt werden konnte. „Für jede einzelne Lehrperson aus unserem Team ist im Mitarbeitenden-Notizbuch ersichtlich, wer wann welche Weiterbildung besucht und sich damit weitere Kompetenzen aneignet" (ebd.). Die Darstellung von Schraner ist sehr überzeugend und ich kann mir gut vorstellen, dass nach der Lektüre einige Schulleitende Schritte in die gleiche Richtung machen möchten. 
Der Beitrag zur Agilität bzw. dem Einführen von OneNote als Kooperationsinstrument hat mich sehr schnell zum Weiterdenken angeregt. Beispielsweise dahin, dass der digitale Wandel an Schulen mehr als die Digitalisierung umfasst. Mit OneNote kommt allenfalls ein neues Medium in die Schule. Je nach Einsatz des Tools verändert dieses aber die Form der Zusammenarbeit an einer Schule. Die Kultur der Digitalität, wie es Felix Stalder nennt, kommt damit deutlich zum Ausdruck und hat durchaus Einfluss auf die Führung und Entwicklung einer Schule. Agile Schulführung geht weiter, als es häufig beschrieben wird, und diese Ausweitung muss zwingend bei einer Einführung von OneNote beachtet werden. Marco Schraner hat, wie ich annehme, dies an seiner Schule beachtet hat, was sich für die Lesenden jedoch nicht sofort erschließt.

Ein zweiter, kritischer Gedanke, der mir beim Lesen des Beitrages in den Sinn kam, war der Umgang mit Transparenz. Transparenz hat, wie wir durch die Digitalisierung sehr deutlich erfahren haben, sowohl positive als auch negative Seiten. Man muss nicht gleich an Foucault denken, aber die beschriebene Transparenz birgt auch die Gefahr der Überwachung. Will ich wirklich, dass alle meine Kolleg*innen - und die Schulleitung? - meine Notizen lesen können? Und habe ich Lust und Zeit, die Notizen meiner Kolleg*innen zu lesen? Mit welcher Absicht lese ich diese? Fragen über Fragen, welche an der Schule von Marco Schraner sicher angesprochen und diskutiert wurden, sich den Lesenden jedoch wiederum nicht von selbst erschließen.

\section{Visionsbuch?}

Schule 21 macht glücklich versteht sich, so zumindest der Untertitel, als Vision des Verbandes der Schulleiterinnen und Schulleiter der Schweiz. Grundsätzlich finde ich es sehr begrüßenswert, dass der Verband eine Vision entwickelt und diese durch ein hohes Maß an Innovation geprägt ist. Das Buch beinhaltet für mich keine Vision, sondern ist im positiven Sinne ein Sammelsurium an interessanten und innovativen Einblicken. Eine Vision müsste deutlich mehr leisten und kann nicht in einem „Book Sprint“ erzeugt werden. Vielleicht wurde der Begriff der Vision aus marketingtechnischen Gründen gewählt und hilft, dem Verband ein modernes und innovatives Gesicht zu geben. Das Buch als Vision zu beschreiben birgt jedoch auch deutlich Gefahren.

Eine der Gefahren ist die fehlende Wissenschaftlichkeit. Wenn wir Schulen innovativ gestalten und die Schüler*innen auf eine uns unbekannte Zukunft hin vorbereiten wollen, dann brauchen wir die Trias von Praxis, Wissenschaft und Politik. Das aus der Praxis geschriebene Buch kann Antrieb und Ausgangspunkt sein, eine Vision zu entwickeln und gemeinsam darüber mit der Politik und Wissenschaft ins Gespräch zu kommen.

Eine zweite Gefahr sehe ich darin, dass nicht alle Passagen im Buch immer überzeugend sind und es auch Aussagen gibt, welche bedenkenswert sind. So schreibt eine Autorin im Buch: „Ja, Sie haben richtig gelesen: Sich der Schülerin und dem Schüler anzupassen ist kein Widerspruch zu starker Führung, sondern der Trick, welcher Türen öffnet" (S. 16). Dass eine Schule 
auch die Aufgabe hat, sich den Lernbedürfnissen der Schüler*innen anzupassen, halte ich für sehr wichtig. Aber in der Führung Tricks anzuwenden, erachte ich als schwierig. Wir sind dann sehr schnell bei der Manipulation und eben gerade nicht beim Ernstnehmen der Schüler*innen. Manche mögen dies als Wortklauberei ansehen. Ich bin überzeugt, dass zur Professionalisierung von Führungspersonen die Frage der Sprache wesentlich ist. Schulleiter*innen tricksen nicht.

Meine kritischen Anmerkungen sind weder Kritik am Text von Marco Schraner noch am Buch, sondern sollen die Grenzen eines solchen Buches aufzeigen. Das Buch gibt interessante und wertvolle Einblicke in innovative Ausschnitte von Schulleitungshandeln. Diese regen an und machen Lust auf (weitere) Entwicklungen. Um die Anregungen an der eigenen Schule aufzunehmen, braucht es aber zwingend eine vertieftere Auseinandersetzung und eine entsprechende Kontextualisierung, denn das Handeln von Schulleiter*innen ist hoch komplex. Das Buch ist gewissermassen das 'Amouse Bouche', welches nicht nährt, aber Lust auf mehr macht.

Ich bin sehr froh und dankbar, dass der Verband der Schulleiterinnen und Schulleiter der Schweiz dieses Buch herausgegeben hat und die zahlreichen Autor*innen Einblicke in ihre Praxis geben. Es ist für mich ein Schnipselbuch, welches sich immer wieder lohnt hervorzuholen und sich davon anregen zu lassen. Daraus eine Vision zu entwickeln, welche die Wissenschaft und Politik einbezieht, halte ich für sehr lohnenswert.

\section{Autor}

Niels Anderegg, MA, MAS

Leiter des Zentrums Management und Leadership an der Pädagogischen Hochschule Zürich. Seine Arbeitsschwerpunkte sind Gemeinschaftliche, agile Schulführung mit Teacher Leadership, Leadership for Learning und Professionalisierung von Führungspersonen von und in Bildungsorganisationen.

Kontakt: niels.anderegg@phzh.ch 